\title{
Enhanced Estimators of Population Variance with the Use of Supplementary Information in Survey Sampling
}

\author{
Showkat Ahmad Lone $\mathbb{D}^{1},{ }^{1}$ Mir Subzar, ${ }^{2}$ and Ankita Sharma $\mathbb{D}^{3}$ \\ ${ }^{1}$ Department of Basic Sciences, Saudi Electronic University, Jeddah Campus, Riyadh, Saudi Arabia \\ ${ }^{2}$ Division of Agricultural Statistics SKUAST-K, Shalimar 190025, India \\ ${ }^{3}$ Department of Statistics, University of Jammu, Jammu, India \\ Correspondence should be addressed to Ankita Sharma; ankitareasi@gmail.com
}

Received 25 March 2021; Revised 11 April 2021; Accepted 16 April 2021; Published 26 April 2021

Academic Editor: Ishfaq Ahmad

Copyright ( 2021 Showkat Ahmad Lone et al. This is an open access article distributed under the Creative Commons Attribution License, which permits unrestricted use, distribution, and reproduction in any medium, provided the original work is properly cited.

\begin{abstract}
In the present study, we propose the proficient class of estimators of the finite population mean, while incorporating the nonconventional location and nonconventional measures of dispersion with coefficient of variation of the auxiliary variable. Properties associated with the suggested class of improved estimators are derived, and an efficiency comparison with the usual unbiased ratio estimator and other existing estimators under consideration in the present study is established. An empirical study has also been provided to validate the theoretical results. Finally, it is established that the proposed class of estimators of the finite population variance proves to be more efficient than the existing estimators mentioned in this study.
\end{abstract}

\section{Introduction}

It is very quite often that utilization of supplementary information in survey sampling which has some sort of strong positive or negative correlation with the response variable is always found to be advantageous. So for the utilization of such information, various methods in survey sampling are presently used to increase precision, with the incorporation of these supplementary information, in estimating the population parameters. Various authors have put their sincere efforts to utilize supplementary information with different sampling designs in different situations in such a way that their estimation procedure becomes more proficient; for details see [1-12]. It is very often that some of the measures are so much affected by extreme observations and can give misleading results. In case of extreme values, using classical methods of estimation provides misleading results, but authors have also put their valuable efforts to come up with solutions to this situation, so that precise results should be obtained even with the presence of outliers in the data. Authors such as Subzar et al. [13] have proposed different robust ratio type estimators in simple random sampling without replacement (SRSWOR) while utilizing the Huber-M estimation technique. Subzar et al. [14] have also proposed different robust ratio type estimators in SRSWOR by utilizing the different robust regression techniques and compared with Ordinary Least Squares (OLS) and Huber-M estimation techniques. Subzar et al. [15] also proposed different robust ratio type estimators by comparing the generalized robust regression techniques with OLS and Huber-M estimation method. Recently, Almanjahie et al. [16] have proposed the generalized class of mean estimators with known measures for outlier's treatment. Also, Shahzad et al. [12] have given a new class of L-Moments-based calibration variance estimators. So in the present study, we made the utilization of nonconventional location parameters, nonconventional measures of dispersion, and their functions with the coefficient of variation of the auxiliary variable in order to suggest the class of estimators for estimating the population variance. The properties of the proposed class of estimators are studied under large sample approximation. It has been shown theoretically as well as empirically that the proposed class of estimators is more efficient than existing estimators mentioned in this study. 


\section{Notations}

Consider a finite population $Z=\left(Z_{1}, Z_{2}, \ldots, Z_{M}\right)$ of $M$ units and let $(z, r)$ be the study and auxiliary variables defined on $Z$ taking values $\left(z_{i}, r_{i}\right)$, respectively, on $Z_{i}(i=1,2, \ldots, M)$. It is desired to estimate the population variance $W_{z}^{2}$ of the study variable $z$ using the information on an auxiliary variable $r$. Let a simple random sample of size $m$ be drawn without replacement from the finite population $Z$. In this paper, we shall ignore the finite population correction (fpc) term. We denote the following.

$\bar{Z}=M^{-1} \sum_{i=1}^{M} z_{i}$ : the population mean of the study variable $z$

$\bar{R}=M^{-1} \sum_{i=1}^{M} r_{i}$ : the population mean of the auxiliary variable $r$

$W_{z}^{2}=(M-1)^{-1} \sum_{i=1}^{M}\left(z_{i}-\bar{Z}\right)^{2}$ : the population mean square/variance of the study variable $z$

$W_{r}^{2}=(M-1)^{-1} \sum_{i=1}^{M}\left(r_{i}-\bar{R}\right)^{2}$ : the population mean square/variance of the auxiliary variable $r$

$\mathrm{TM}=\left(Q_{1}+2 Q_{2}+Q_{3} / 4\right)$ : tri-mean of the auxiliary variable $r$

$\mathrm{HL}=$ median $\left(\left(R_{j}+R_{k}\right) / 2,1 \leq j \leq k \leq M\right)$ : Hodges-Lehmann estimator of the auxiliary variable $r$

$\mathrm{MR}=\left(R_{(1)}+R_{(M)} / 2\right)$ : mid-range of the auxiliary variable $r$

$G=(4 / M-1) \sum_{i=1}^{M}(2 i-M-1 / 2 M) R_{(i)}$ : Gini's mean difference of the auxiliary variable $r$

$D=(2 \sqrt{\pi} / M(M-1)) \sum_{i=1}^{M}(i-(M+1 / 2)) R_{(i)}$ : Downton's method of the auxiliary variable $r$

$S_{\mathrm{pw}}=\left(\sqrt{\pi} / M^{2}\right) \sum_{i=1}^{M}(2 i-M-1) R_{(i)}$ : probability weighted moments of the auxiliary variable $r$ :

$$
\begin{aligned}
& \mu_{40}^{*}=\left(\mu_{40}-1\right), \\
& \mu_{04}^{*}=\left(\mu_{04}-1\right), \\
& \mu_{22}^{*}=\left(\mu_{22}-1\right),
\end{aligned}
$$

where $\mu_{r s}=\lambda_{b c} /\left(\lambda_{02}^{c / 2} \lambda_{20}^{b / s}\right), \lambda_{r s}=M^{-1} \sum_{i=1}^{M}\left(z_{i}-\bar{Z}\right)^{b}\left(r_{i}-\bar{R}\right)^{c}$, and $(b, c)$ are nonnegative integers.

\section{The Proposed Class of Estimators}

In the present study, we have made the incorporation of nonconventional location parameters, nonconventional measures of dispersion, and their function with the coefficient of variation, whose generalized class for estimating population variance is given as

$$
l=\left[\alpha_{1} w_{z}^{2}+\alpha_{2}\left(W_{r}^{2}-w_{r}^{2}\right)\right]\left(\frac{\gamma W_{r}^{2}+\varphi Q^{2}}{\gamma w_{r}^{2}+\varphi Q^{2}}\right),
$$

where $\left(\gamma W_{r}^{2}+\varphi Q^{2}\right)>0,\left(\gamma w_{r}^{2}+\varphi Q^{2}\right)>0$, and $(\gamma, Q)$ are either real constants or functions of known parameters of an auxiliary variable $r$ and $\varphi$ is a constant such that $|\varphi| \leq 1$ which is a more flexible condition as given by Singh et al. [5] and
Solanki et al. [17] over the constant $\varphi$ in their estimators, and $\left(\alpha_{1}, \alpha_{2}\right)$ are constants such that the mean squared error (MSE) is minimum. Here, we note that $0 \leq \varphi \leq 1$. For suitable values of $\left(\alpha_{1}, \alpha_{2}, \gamma, \varphi\right)$, the proposed class of estimators "l" reduces to some known existing estimators based on quartiles and their functions given in Table 1.

From (2), we would like to remark the suitable values of $\left(\alpha_{1}, \alpha_{2}, \gamma, \varphi\right)$ and one can generate different estimators. For example, we have developed some new estimators from the proposed class of estimators "l" which are listed in Table 2.

While obtaining the expression of bias and mean square error (MSE) for the suggested class of estimator "l", we write

$$
\begin{aligned}
& w_{z}^{2}=W_{z}^{2}\left(1+\vartheta_{0}\right), \\
& w_{r}^{2}=W_{r}^{2}\left(1+\vartheta_{1}\right),
\end{aligned}
$$

such that $E\left(\vartheta_{0}\right)=E\left(\vartheta_{1}\right)=0$, and up to the first order of approximation, while fpc term is ignored, we have

$$
\begin{array}{r}
E\left(\vartheta_{0}^{2}\right)=\left(\frac{\mu_{40}^{*}}{m}\right), \\
E\left(\vartheta_{1}^{2}\right)=\left(\frac{\mu_{04}^{*}}{m}\right), \\
E\left(\vartheta_{0} \vartheta_{1}\right)=\left(\frac{\mu_{22}^{*}}{m}\right) .
\end{array}
$$

Now, in terms of $\vartheta^{\prime} s$, the estimator " $l$ ", given in (2), is expressed as

$$
l=W_{z}^{2}\left[\alpha_{1}\left(1+\vartheta_{0}\right)-\alpha_{2} b \vartheta_{1}\right]\left(1+\theta^{*} \vartheta_{1}\right)^{-1},
$$

where

$$
\begin{aligned}
b & =\frac{W_{r}^{2}}{W_{z}^{2}}, \\
\theta^{*} & =\frac{\gamma W_{r}^{2}}{\left(\gamma W_{r}^{2}+\varphi Q^{2}\right)} .
\end{aligned}
$$

We assume that $\left|\theta^{*} \vartheta_{1}\right|<1$, so that $\left(1+\theta^{*} \vartheta_{1}\right)^{-1}$ is expendable. Expanding the right-hand side of (5) and multiplying out, we have

$$
\begin{aligned}
\theta \cong & W_{z}^{2}\left[\alpha_{1}\left(1+\vartheta_{0}-\theta^{*} \vartheta_{1}-\theta^{*} \vartheta_{0} \vartheta_{1}+\theta^{* 2} \vartheta_{1}^{2}\right)\right. \\
& \left.+\alpha_{2} b\left(\theta^{*} \vartheta_{1}^{2}-\vartheta_{1}\right)\right] \\
\text { or }\left(\theta-W_{z}^{2}\right) \cong & W_{z}^{2}\left[\alpha_{1}\left(1+\vartheta_{0}-\theta^{*} \vartheta_{1}-\theta^{*} \vartheta_{0} \vartheta_{1}+\theta^{* 2} \vartheta_{1}^{2}\right)\right. \\
& \left.+\alpha_{2} b\left(\theta^{*} \vartheta_{1}^{2}-\vartheta_{1}\right)-1\right] .
\end{aligned}
$$

Taking expectation of both sides of (7), we get the bias of the estimators $l$ to the first degree of approximation as

$$
B(l)=W_{z}^{2}\left[\alpha_{1}\left\{1+\left(\frac{\theta^{*}}{m}\right)\left(\theta^{*} \mu_{04}^{*}-\mu_{22}^{*}\right)\right\}+\alpha_{2}\left(\frac{b \theta^{*}}{m}\right) \mu_{04}^{*}-1\right] .
$$


TABle 1: Some existing known estimators of the class of estimators $l$.

\begin{tabular}{|c|c|c|c|c|c|c|}
\hline \multirow{2}{*}{ S. no. } & \multirow{2}{*}{ Estimators } & \multicolumn{5}{|c|}{ Values of constants } \\
\hline & & $\alpha_{1}$ & $\alpha_{2}$ & $\gamma$ & Q & $\varphi$ \\
\hline 1 & $l_{1}=w_{z}^{2}$, usual unbiased estimator & 1 & 0 & 0 & - & - \\
\hline 2 & $l_{2}=w_{z}^{2}\left(W_{r}^{2} / w_{r}^{2}\right)$, Das and Tripathi [1] and Isaki [4] estimator & 1 & 0 & 1 & - & 0 \\
\hline 3 & $l_{3}=w_{z}^{2}\left(W_{r}^{2}+\varphi Q_{1}^{2} / w_{r}^{2}+\varphi Q_{1}^{2}\right)$, Singh et al. [5] estimator & 1 & 0 & 1 & $Q_{1}$ & $\delta$ \\
\hline 4 & $l_{4}=w_{z}^{2}\left(W_{r}^{2}+\varphi Q_{2}^{2} / w_{r}^{2}+\varphi Q_{2}^{2}\right)$, Singh et al. [5] estimator & 1 & 0 & 1 & $Q_{2}$ & $\delta$ \\
\hline 5 & $l_{5}=w_{z}^{2}\left(W_{r}^{2}+\varphi Q_{3}^{2} / w_{r}^{2}+\varphi Q_{3}^{2}\right)$, Singh et al. [5] estimator & 1 & 0 & 1 & $Q_{3}$ & $\delta$ \\
\hline 6 & $l_{6}=w_{z}^{2}\left(W_{r}^{2}+\varphi Q_{r}^{2} / w_{r}^{2}+\varphi Q_{r}^{2}\right)$, Singh et al. [5] estimator & 1 & 0 & 1 & $Q_{r}$ & $\delta$ \\
\hline 7 & $l_{7}=w_{z}^{2}\left(W_{r}^{2}+\varphi Q_{d}^{2} / w_{r}^{2}+\varphi Q_{d}^{2}\right)$, Singh et al. [5] estimator & 1 & 0 & 1 & $Q_{d}$ & $\delta$ \\
\hline 8 & $l_{8}=w_{z}^{2}\left(W_{r}^{2}+\varphi Q_{a}^{2} / w_{r}^{2}+\varphi Q_{a}^{2}\right)$, Singh et al. [5] estimator & 1 & 0 & 1 & $Q_{a}$ & $\delta$ \\
\hline 9 & $l_{9}=w_{z}^{2}\left(C_{r} W_{r}^{2}+\varphi Q_{1}^{2} / C_{r} w_{r}^{2}+\varphi Q_{1}^{2}\right)$, Solanki et al. [17] estimator & 1 & 0 & $C_{r}$ & $Q_{1}$ & $\delta$ \\
\hline 10 & $l_{10}=w_{z}^{2}\left(\beta_{2(r)} W_{r}^{2}+\varphi Q_{2}^{2} / \beta_{2(r)} s_{r}^{2}+\varphi Q_{2}^{2}\right)$, Solanki et al. [17] estimator & 1 & 0 & $\beta_{2(r)}$ & $Q_{2}$ & $\delta$ \\
\hline 11 & $l_{11}=w_{z}^{2}\left(\bar{R} W_{r}^{2}+\varphi Q_{3}^{2} / \bar{R} w_{r}^{2}+\varphi Q_{3}^{2}\right)$, Solanki et al. [17] estimator & 1 & 0 & $\bar{R}$ & $Q_{3}$ & $\delta$ \\
\hline 12 & $l_{12}=w_{z}^{2}\left(\beta_{2(r)} W_{r}^{2}+\varphi Q_{r}^{2} / \beta_{2(r)} w_{r}^{2}+\varphi Q_{r}^{2}\right)$, Solanki et al. [17] estimator & 1 & 0 & $\beta_{2(r)}$ & $Q_{r}$ & $\delta$ \\
\hline 13 & $l_{13}=w_{z}^{2}\left(\rho W_{r}^{2}+\varphi Q_{d}^{2} / \rho w_{r}^{2}+\varphi Q_{d}^{2}\right)$, Solanki et al. [17] estimator & 1 & 0 & $\rho$ & $Q_{d}$ & $\delta$ \\
\hline 14 & $l_{14}=w_{z}^{2}\left(\rho W_{r}^{2}+\varphi Q_{a}^{2} / \rho w_{z}^{2}+\varphi Q_{a}^{2}\right)$, Solanki et al. [17] estimator & 1 & 0 & $\rho$ & $Q_{a}$ & $\delta$ \\
\hline
\end{tabular}

TABLE 2: Some new members of the class of estimators $l$.

\begin{tabular}{|c|c|c|c|c|c|c|}
\hline \multirow{2}{*}{ S. no. } & \multirow{2}{*}{ Estimators } & \multicolumn{5}{|c|}{ Values of constant } \\
\hline & & $w_{1}$ & $w_{2}$ & $\gamma$ & Q & $\varphi$ \\
\hline 15 & $l_{15}=\left[\alpha_{1} w_{z}^{2}+\alpha_{2}\left(W_{r}^{2}-w_{r}^{2}\right)\right]\left(\gamma W_{r}^{2}+\varphi T M^{2} / \gamma w_{r}^{2}+\varphi T M^{2}\right)$ & $\alpha_{1}$ & $\alpha_{2}$ & $C_{r}$ & TM & $\varphi$ \\
\hline 16 & $l_{16}=\left[\alpha_{1} w_{z}^{2}+\alpha_{2}\left(W_{r}^{2}-w_{r}^{2}\right)\right]\left(\gamma W_{r}^{2}+\varphi M R^{2} / \gamma w_{r}^{2}+\varphi M R^{2}\right)$ & $\alpha_{1}$ & $\alpha_{2}$ & $C_{r}$ & $\mathrm{MR}$ & $\varphi$ \\
\hline 17 & $l_{17}=\left[\alpha_{1} w_{z}^{2}+\alpha_{2}\left(W_{r}^{2}-w_{r}^{2}\right)\right]\left(\gamma W_{r}^{2}+\varphi H L^{2} / \gamma w_{r}^{2}+\varphi H L^{2}\right)$ & $\alpha_{1}$ & $\alpha_{2}$ & $C_{r}$ & $\mathrm{HL}$ & $\varphi$ \\
\hline 18 & $l_{18}=\left[\alpha_{1} w_{z}^{2}+\alpha_{2}\left(W_{r}^{2}-w_{r}^{2}\right)\right]\left(\gamma W_{r}^{2}+\varphi G^{2} / \gamma w_{r}^{2}+\varphi G^{2}\right)$ & $\alpha_{1}$ & $\alpha_{2}$ & $C_{r}$ & $G$ & $\varphi$ \\
\hline 19 & $l_{19}=\left[\alpha_{1} w_{z}^{2}+\alpha_{2}\left(W_{r}^{2}-w_{r}^{2}\right)\right]\left(\gamma W_{r}^{2}+\varphi D^{2} / \gamma w_{r}^{2}+\varphi D^{2}\right)$ & $\alpha_{1}$ & $\alpha_{2}$ & $C_{r}$ & $D$ & $\varphi$ \\
\hline 20 & $l_{20}=\left[\alpha_{1} w_{z}^{2}+\alpha_{2}\left(W_{r}^{2}-w_{r}^{2}\right)\right]\left(\gamma W_{r}^{2}+\varphi S_{\mathrm{pw}}^{2} / \gamma w_{r}^{2}+\varphi S_{\mathrm{pw}}^{2}\right)$ & $\alpha_{1}$ & $\alpha_{2}$ & $C_{r}$ & $S_{\mathrm{pw}}$ & $\varphi$ \\
\hline
\end{tabular}

Squaring both sides of (7), neglecting terms of $\vartheta^{\prime} s$ having the power greater than two, we have

$$
\begin{aligned}
\left(\theta-W_{z}^{2}\right)^{2} \cong & W_{z}^{4}\left[1+\alpha_{1}^{2}\left(1+2 \vartheta_{0}-2 \theta^{*} \vartheta_{1}+\vartheta_{0}^{2}+3 \theta^{* 2} \vartheta_{1}^{2}-4 \theta^{*} \vartheta_{0} \vartheta_{1}\right)+\alpha_{2}^{2} b^{2} \vartheta_{1}^{2}\right. \\
& +2 \alpha_{1} \alpha_{2} b\left(2 \theta^{*} \vartheta_{1}^{2}-\vartheta_{0} \vartheta_{1}-\vartheta_{1}\right)-2 \alpha_{1}\left(1+\vartheta_{0}-\theta^{*} \vartheta_{1}-\theta^{*} \vartheta_{0} \vartheta_{1}+\theta^{* 2} \vartheta_{1}\right) \\
& \left.-2 \alpha_{2} b\left(\theta^{*} \vartheta_{1}^{2}-\vartheta_{1}\right)\right]
\end{aligned}
$$

Taking expectation of both sides of (9), we get the MSE of the estimator $l$ to the first degree of approximation (ignoring fpc term) as

$$
\operatorname{MSE}(l)=W_{z}^{4}\left[1+\alpha_{1}^{2} d_{1}+\alpha_{2}^{2} d_{2}+2 \alpha_{1} \alpha_{2}-2 \alpha_{1} d_{4}-2 \alpha_{2} d_{5}\right],
$$

where 


$$
\begin{aligned}
& d_{1}=\left[1+\frac{1}{m}\left(\mu_{40}^{*}+3 \theta^{* 2} \mu_{04}^{*}-4 \theta^{*} \mu_{22}^{*}\right)\right], \\
& d_{2}=\frac{b^{2} \mu_{04}^{*}}{m} \\
& d_{3}=\left(\frac{b}{m}\right)\left(2 \theta^{*} \mu_{04}^{*}-\mu_{22}^{*}\right), \\
& d_{4}=\left[1+\frac{\theta^{*}}{m}\left(\theta^{*} \mu_{04}^{*}-\mu_{22}^{*}\right)\right] \\
& d_{5}=\frac{b \theta^{*}}{m} \mu_{04}^{*} .
\end{aligned}
$$

Differentiating MSE $(l)$ with respect to $\alpha_{1}$ and $\alpha_{2}$ and equating them to zero, we have

$$
\left[\begin{array}{ll}
d_{1} & d_{3} \\
d_{3} & d_{2}
\end{array}\right]\left[\begin{array}{l}
\alpha_{1} \\
\alpha_{2}
\end{array}\right]=\left[\begin{array}{l}
d_{4} \\
d_{5}
\end{array}\right]
$$
as

Simplifying (12), we get the optimum values of $\alpha_{1}$ and $\alpha_{2}$

$$
\begin{aligned}
& \alpha_{1}=\frac{\left(d_{2} d_{4}-d_{3} d_{5}\right)}{\left(d_{1} d_{2}-d_{3}^{2}\right)}=\alpha_{10} \text { (say), } \\
& \alpha_{2}=\frac{\left(d_{1} d_{5}-d_{3} d_{4}\right)}{\left(d_{1} d_{2}-d_{3}^{2}\right)}=\alpha_{20} \text { (say). }
\end{aligned}
$$

Inserting (13) in (10), we get the resulting minimum MSE of "l" given by

$$
\min \cdot \operatorname{MSE}(l)=W_{z}^{4}\left[1-\frac{\left(d_{2} d_{4}^{2}-2 d_{3} d_{4} d_{5}+d_{1} d_{5}^{2}\right)}{\left(d_{1} d_{2}-d_{3}^{2}\right)}\right] .
$$

Thus we establish the following theorem.

Theorem 1. To the first degree of approximation

$$
\operatorname{MSE}(l) \geq W_{z}^{4}\left[1-\frac{\left(d_{2} d_{4}^{2}-2 d_{3} d_{4} d_{5}+d_{1} d_{5}^{2}\right)}{\left(d_{1} d_{2}-d_{3}^{2}\right)}\right],
$$

with equality holding if

$$
\begin{aligned}
& \alpha_{1}=\alpha_{10}, \\
& \alpha_{2}=\alpha_{20},
\end{aligned}
$$

where $\alpha_{i 0}^{\prime} s(i=1,2)$ are given in (13).

Special Case 1: for $\alpha_{1}=1$ in (2), we get an alternative class of estimators for the population variance $W_{z}^{2}$ as

$$
t^{*}=\left[w_{z}^{2}+\alpha_{2}\left(W_{r}^{2}-w_{r}^{2}\right)\right]\left(\frac{\gamma W_{r}^{2}+\varphi Q^{2}}{\gamma w_{r}^{2}+\varphi Q^{2}}\right) .
$$

Putting $\alpha_{1}=0$ in (8) and (10), we get the bias and MSE of $l^{*}$ to the first degree of approximation, given by

$$
\begin{aligned}
B\left(l^{*}\right) & =\left(\frac{W_{z}^{2}}{m}\right) \theta^{*}\left[\left(\theta^{*}+b\right) \mu_{04}^{*}-\mu_{22}^{*}\right], \\
\operatorname{MSE}\left(l^{*}\right) & =W_{z}^{4}\left[1+d_{1}-2 d_{4}+\alpha_{2}^{2} d_{2}-2 \alpha_{2}\left(d_{5}-d_{3}\right)\right] .
\end{aligned}
$$

The $\operatorname{MSE}\left(l^{*}\right)$ is minimum when

$$
\alpha_{20}^{*}=\frac{\left(d_{5}-d_{3}\right)}{\left(d_{2}\right)} \text {. }
$$

Thus, the resulting minimum MSE of $\left(l^{*}\right)$ is given by

$$
\begin{aligned}
\min \cdot \operatorname{MSE}\left(l^{*}\right) & =W_{z}^{4}\left[1+d_{1}-2 d_{4}-\frac{\left(d_{5}-d_{3}\right)^{2}}{d_{2}}\right] \\
& =\left(\frac{W_{z}^{4}}{m}\right)\left[\mu_{40}^{*}-\frac{\mu_{22}^{* 2}}{\mu_{04}^{*}}\right] .
\end{aligned}
$$

This equals to the minimum MSE of the difference estimator $l_{d}$ envisaged by [1]

$$
l_{d}=w_{z}^{2}+\alpha_{2}\left(W_{r}^{2}-w_{r}^{2}\right) .
$$

Special Case 2: for $\alpha_{2}=0$ in (2), we get another class of estimators for population variance $W_{z}^{2}$ as

$$
l_{1}^{*}=\left[\alpha_{1} w_{z}^{2}\right]\left(\frac{\gamma W_{r}^{2}+\varphi Q^{2}}{\gamma w_{r}^{2}+\varphi Q^{2}}\right) .
$$

Putting $\alpha_{2}=0$ in (8) and (10), we get the bias and MSE of $l_{1}^{*}$, respectively, as

$$
\begin{aligned}
B\left(l^{*}\right) & =W_{z}^{2}\left[\alpha_{1}\left\{1+\frac{\theta^{*}}{m}\left(\theta^{*} \mu_{04}^{*}-\mu_{22}^{*}\right)\right\}-1\right], \\
\operatorname{MSE}\left(l^{*}\right) & =W_{z}^{4}\left[1+\alpha_{1}^{2} d_{1}-2 \alpha_{1} d_{4}\right] .
\end{aligned}
$$

The $\operatorname{MSE}\left(l^{*}\right)$ is minimum for

$$
\alpha_{10}^{*}=\frac{d_{4}}{d_{1}}
$$

Thus, the resulting minimum MSE of $l^{*}$ is given by

$$
\min \cdot \operatorname{MSE}\left(l^{*}\right)=W_{z}^{4}\left[1-\frac{d_{4}^{2}}{d_{1}}\right]
$$

Thus, we state the following corollary.

Corollary 1. To the first degree of approximation

$$
\operatorname{MSE}\left(l_{1}^{*}\right) \geq W_{z}^{4}\left[1-\frac{d_{4}^{2}}{d_{1}}\right],
$$

with equality holding if

$$
\alpha_{1}=\alpha_{10}^{*}=\frac{d_{4}}{d_{1}} .
$$


Special Case 3: if we set $\left(\alpha_{1}, \alpha_{2}\right)=(1,0)$ in (2), the class of estimators $l$ reduces to the estimator

$$
l_{1}^{* *}=w_{z}^{2}\left(\frac{\gamma W_{r}^{2}+\varphi Q^{2}}{\gamma w_{r}^{2}+\varphi Q^{2}}\right)
$$

which includes Solanki et al. [17] estimators $l_{9}$ to $l_{14}$.

Putting $\left(\alpha_{1}, \alpha_{2}\right)=(1,0)$ in (8) and (10), we get the bias and MSE of $l_{1}^{* *}$ to the first degree of approximation (ignoring fpc term), respectively, as

$$
\begin{aligned}
& B\left(l_{1}^{* *}\right)=\left(\frac{W_{z}^{2} \theta^{*}}{m}\right)\left(\theta^{*} \mu_{04}^{*}-\mu_{22}^{*}\right), \\
& \operatorname{MSE}\left(l_{1}^{* *}\right)=W_{z}^{4}\left[1+d_{1}-2 d_{4}\right], \\
& =\left(\frac{W_{z}^{4}}{m}\right)\left(\mu_{40}^{*}+\theta^{* 2} \mu_{04}^{*}-2 \theta^{*} \mu_{22}^{*}\right) .
\end{aligned}
$$

Special Case 4: if we set $\left(\alpha_{1}, \alpha_{2}, \gamma\right)=\left(\alpha_{1}, 0,1\right)$ in (2), the class of estimators $l$ reduces to the class of estimators of $W_{z}^{2}$ as

$$
l_{2}^{*}=\alpha_{1} w_{z}^{2}\left(\frac{W_{r}^{2}+\varphi Q^{2}}{w_{r}^{2}+\varphi Q^{2}}\right)
$$

Inserting $\left(\alpha_{1}, \alpha_{2}, \gamma\right)=\left(\alpha_{1}, 0,1\right)$ in (8) and (10), we get the bias and MSE of $l_{2}^{*}$ to the first degree of approximation (ignoring $\mathrm{fpc}$ term), respectively, as

$$
\begin{aligned}
B\left(l_{2}^{*}\right) & =W_{z}^{2}\left[\alpha_{1}\left\{1+\left(\frac{\theta}{m}\right)\left(\theta \mu_{04}^{*}-\mu_{22}^{*}\right)\right\}-1\right], \\
\operatorname{MSE}\left(l_{2}^{*}\right) & =W_{z}^{4}\left[1+\alpha_{1}^{2} d_{1}^{*}-\alpha_{1} d_{4}^{*}\right],
\end{aligned}
$$

where

$$
\begin{aligned}
d_{1}^{*} & =\left[1+\frac{1}{m}\left(\mu_{40}^{*}+3 \theta^{2} \mu_{04}^{*}-4 \theta \mu_{22}^{*}\right)\right], \\
d_{4}^{*} & =\left[1+\frac{\theta}{m}\left(\theta \mu_{04}^{*}-\mu_{22}^{*}\right)\right], \\
\theta & =\frac{W_{r}^{2}}{W_{r}^{2}+\phi Q^{2}} .
\end{aligned}
$$

The $\operatorname{MSE}\left(l_{2}^{*}\right)$ at $(34)$ is minimized for

$$
\alpha_{10}^{* *}=\frac{d_{4}^{*}}{d_{1}^{*}} .
$$

Substitution of (36) in (34) yields the minimum MSE of $l_{2}^{*}$ and is given by

$$
\min \cdot \operatorname{MSE}\left(l_{2}^{*}\right)=W_{z}^{4}\left[1-\frac{d_{4}^{* 2}}{d_{1}^{*}}\right] .
$$

Thus, we established the following corollary.

Corollary 2. To the first degree of approximation

$$
\operatorname{MSE}\left(l_{2}^{*}\right) \geq W_{z}^{4}\left[1-\frac{d_{4}^{* 2}}{d_{1}^{*}}\right],
$$

with equality holdings if

$$
\alpha_{1}=\alpha_{10}^{* *}=\frac{d_{4}^{*}}{d_{1}^{*}}
$$

Special Case 5: for $\left(\alpha_{1}, \alpha_{2}, \gamma\right)=(1,0,1)$ in (2), the class of estimators " $l$ " reduces to the class of estimators of $W_{z}^{2}$ as

$$
l_{2}^{* *}=w_{z}^{2}\left(\frac{W_{r}^{2}+\varphi Q^{2}}{w_{r}^{2}+\varphi Q^{2}}\right)
$$

which includes Singh et al. [5] estimators $l_{3}$ to $l_{8}$.

Inserting $\left(\alpha_{1}, \alpha_{2}, \gamma\right)=(1,0,1)$ in (8) and (10), we get the bias and MSE of $l_{2}^{* *}$ to the first degree of approximation (ignoring $\mathrm{fpc}$ term), respectively, given by

$$
\begin{aligned}
l_{2}^{* *} & =\left(\frac{W_{z}^{2} \theta^{*}}{m}\right)\left(\theta^{*} \mu_{04}^{*}-\mu_{22}^{*}\right), \\
\operatorname{MSE}\left(l_{2}^{* *}\right) & =W_{z}^{4}\left[1+d_{1}^{*}-2 d_{4}^{*}\right] \\
& =\left(\frac{W_{z}^{4}}{m}\right)\left(\mu_{40}^{*}+\theta^{2} \mu_{04}^{*}-2 \theta \mu_{22}^{*}\right) .
\end{aligned}
$$

\section{Efficiency Comparison}

To the first degree of approximation, the MSE of $w_{z}^{2}$ and $l_{2}$ are, respectively, given by

$$
\begin{aligned}
& \operatorname{MSE}\left(w_{z}^{2}\right)=\left(\frac{W_{z}^{4}}{m}\right) \mu_{40}^{*}, \\
& \operatorname{MSE}\left(l_{2}\right)=\left(\frac{W_{z}^{4}}{m}\right)\left(\mu_{40}^{*}+\mu_{04}^{*}+2 \mu_{22}^{*}\right) .
\end{aligned}
$$

From (20), (43), and (44), we have

$$
\begin{aligned}
& \operatorname{MSE}\left(w_{z}^{2}\right)-\min \cdot \operatorname{MSE}\left(l^{*} \text { or } l_{d}\right)=\left(\frac{W_{z}^{4} \mu_{22}^{* 2}}{m \mu_{04}^{*}}\right)>0, \\
& \operatorname{MSE}\left(l_{2}\right)-\min \cdot \operatorname{MSE}\left(l_{2}^{*}\right)=\frac{W_{z}^{4}\left(\mu_{04}^{*}-\mu_{22}^{*}\right)^{2}}{m \mu_{04}^{*}} \geq 0 .
\end{aligned}
$$

It follows from (45) and (46) that the proposed class of estimators $l^{*}$ or difference estimator $l_{d}$ is better than the usual unbiased estimator $w_{z}^{2}$ and the ratio type estimator $l_{2}$ due to $[1,4]$.

From (37) and (42), we have

$$
\operatorname{MSE}\left(l_{2}^{* *}\right)-\min \cdot \operatorname{MSE}\left(l_{2}^{*}\right)=\frac{W_{z}^{4}\left(d_{1}^{*}-d_{4}^{*}\right)^{2}}{d_{1}^{*}} \geq 0 .
$$

This follows that the proposed class of estimators $l_{2}^{*}$ is more efficient than the proposed class of estimators $l_{2}^{* *}$ according to Singh et al. [5]. Thus, the estimator $l_{2}^{*}$ is an improvement over the Singh et al. [5] estimators $l_{2}^{* *}$. 
TABLE 3: The parameters of population data set.

\begin{tabular}{lccccccc}
\hline$M$ & 80 & $C_{z}$ & 0.3542 & $Q_{1}$ & 5.1500 & TM & 9.318 \\
$m$ & 20 & $W_{r}$ & 8.4563 & $Q_{2}$ & 10.300 & MR & 10.405 \\
$\bar{Z}$ & 51.8264 & $C_{r}$ & 0.7507 & $Q_{3}$ & 16.975 & HL & 17.955 \\
$\bar{R}$ & 11.2646 & $\mu_{04}$ & 2.8664 & $Q_{r}$ & 11.825 & 9.0408 \\
$\rho$ & 0.9413 & $\mu_{40}$ & 2.2667 & $Q_{d}$ & 5.9125 & $D$ & 8.0138 \\
$W_{z}$ & 18.3569 & $\mu_{22}$ & 2.2209 & $Q_{a}$ & 11.0625 & $S_{\mathrm{pw}}$ & 7.9136 \\
\hline
\end{tabular}

TABLE 4: PRE of estimators $l_{k}(k=15,16, \ldots, 20)$ with respect to $l_{1}=w_{z}^{2}$.

\begin{tabular}{|c|c|c|c|c|c|c|}
\hline \multirow{2}{*}{$\phi$} & \multicolumn{6}{|c|}{$\operatorname{PRE}\left(w_{z}^{2}\right)$} \\
\hline & $l_{15}$ & $l_{16}$ & $l_{17}$ & $l_{18}$ & $l_{19}$ & $l_{20}$ \\
\hline-1.00 & 257.0391 & 259.3591 & 291.8213 & 258.2640 & 256.4163 & 253.0021 \\
\hline-0.75 & 255.2481 & 257.2658 & 275.3213 & 256.0011 & 253.9286 & 252.8256 \\
\hline-0.50 & 254.2359 & 256.9329 & 273.3492 & 255.3651 & 253.6165 & 252.4152 \\
\hline-0.25 & 253.9139 & 256.1231 & 273.1029 & 254.9606 & 253.0259 & 252.0081 \\
\hline 0.00 & 253.1029 & 255.8569 & 273.0051 & 254.0436 & 252.8169 & 251.5621 \\
\hline 0.25 & 252.9320 & 255.0091 & 272.9321 & 253.8186 & 252.0411 & 251.0561 \\
\hline 0.50 & 253.1001 & 254.8563 & 272.3649 & 253.5646 & 251.9103 & 250.3961 \\
\hline 0.75 & 254.2569 & 254.6561 & 272.1536 & 253.3963 & 251.3851 & 250.0011 \\
\hline 1.00 & 254.2031 & 254.5341 & 271.9806 & 252.8646 & 251.0091 & 249.5651 \\
\hline
\end{tabular}

From (25) and (30), we have

$$
\operatorname{MSE}\left(l_{1}^{* *}\right)-\min \cdot \operatorname{MSE}\left(l_{1}^{*}\right)=\frac{W_{z}^{4}\left(d_{1}-d_{4}\right)^{2}}{d_{1}} \geq 0
$$

Expression (48) clearly indicates that the proposed class of estimators $l_{1}^{*}$ is better than class of estimator $l_{1}^{* *}$ recently proposed by Solanki et al. [17]. Thus, the estimators $l_{1}^{*}$ are an improvement over the estimator $l_{1}^{* *}$ due to Solanki et al. [17].

Furthermore, from (14) and (20), we have

$$
\min \cdot \operatorname{MSE}\left(l^{*}\right)-\min \cdot \operatorname{MSE}(l)=\frac{W_{z}^{4}\left[d_{2}\left(d_{1}-d_{4}\right)+d_{3}\left(d_{5}-d_{3}\right)\right]^{2}}{d_{2}\left(d_{1} d_{2}-d_{3}^{2}\right)}>0
$$

It follows from (49) that the proposed class of estimators " $l$ " is better than the class of estimators $l^{*}$ and the difference estimator $l_{d}$.

Next, from (14) and (25), we have

$$
\min \cdot \operatorname{MSE}\left(l_{1}^{*}\right)-\min \cdot \operatorname{MSE}(l)=\frac{W_{z}^{4}\left(d_{1} d_{5}-d_{3} d_{4}\right)^{2}}{d_{1}\left(d_{1} d_{2}-d_{3}^{2}\right)} \geq 0
$$

Thus, from (48) and (50), we have the following inequality:

$$
\min \cdot \operatorname{MSE}(l) \leq \min \cdot \operatorname{MSE}\left(l_{1}^{*}\right) \leq \min \cdot \operatorname{MSE}\left(l_{1}^{* *}\right) .
$$

This clearly shows that the proposed family of estimators $l$ is better than families of estimators $l_{1}^{*}$ and $l_{1}^{* *}$.

Finally, from the above theoretical comparisons we conclude that the proposed family of estimators $l$ is better than the usual unbiased estimator $l_{1}=w_{z}^{2}$, [4] ratio type estimator $l_{2}$, and the estimators $\left(l_{3}\right.$ to $\left.l_{8}\right)$ and $\left(l_{9}\right.$ to $\left.l_{14}\right)$ recently proposed by Singh et al. [5] and Solanki et al. [17], respectively, and for other families of estimators $\left(l_{1}^{*}, l_{2}^{*}\right)$.

\section{Empirical Study}

The performances of the proposed estimators $l_{k}(k=15,16, \ldots, 20)$, which are members of the suggested class of estimators, $l$, are evaluated against the usual unbiased estimators $w_{z}^{2}$ and the estimators $l_{i}(i=2,3, \ldots, 14)$ proposed by $[1,4,5,10]$ for the population data set (source: [18]) summarized in Table 3.

We have computed the percent relative efficiencies (PREs) of the suggested estimators $l_{k}(k=15,16, \ldots, 20)$ with respect to the usual unbiased estimator $w_{z}^{2}$ in the range of $\varphi \in(-1.0,1.0)$ by using the following formula:

$$
\begin{aligned}
\operatorname{PRE}\left(l_{k}, w_{z}^{2}\right) & =\frac{\operatorname{MSE}\left(w_{z}^{2}\right)}{\operatorname{MSE}\left(l_{k}\right)} \times 100 \\
& =\frac{\mu_{40}^{*}}{m\left[1-\left(d_{1} d_{4}^{2}-2 d_{3} d_{4} d_{5}+\left(d_{1} d_{3}^{2} / d_{1} d_{2}\right)-d_{3}^{2}\right)\right]} \times 100,
\end{aligned}
$$

and the findings are summarized in Table 4. 
TABLE 5: PREs of the estimators $l_{1}=w_{z}^{2}, l_{2}$ and $l_{i}(i=3, \ldots, 14)$.

\begin{tabular}{lcc}
\hline Estimator & Value of $\varphi$ & PRE $\left(w_{z}^{2}\right)$ \\
\hline$l_{1}$ & - & 100.00 \\
$l_{2}$ & - & 183.23 \\
$l_{3}$ & 1.00 & 209.67 \\
$l_{4}$ & 0.50 & 230.60 \\
$l_{5}$ & 0.30 & 230.28 \\
$l_{6}$ & 0.70 & 230.57 \\
$l_{7}$ & 1.00 & 200.87 \\
$l_{8}$ & 0.60 & 230.80 \\
$l_{9}$ & 1.00 & 270.38 \\
$l_{10}$ & 270.61 \\
$l_{11}$ & 1.00 & 263.50 \\
$l_{12}$ & 1.00 & 270.58 \\
$l_{13}$ & 0.80 & 270.61 \\
$l_{14}$ & 1.00 & 270.58 \\
\hline
\end{tabular}

For the purpose of comparison of the proposed estimators $l_{15}$ to $l_{20}$ with that of the usual unbiased estimator $l_{1}=w_{z}^{2}$, [4] estimator $l_{2}$, [5] estimators $\left(l_{3}\right.$ to $\left.l_{8}\right)$, and [17] estimators $\left(l_{9}\right.$ to $\left.l_{14}\right)$, in Table 5 , we give the PREs of the estimators $l_{1}, l_{2}$ and the PREs of the estimators $l_{3}$ to $l_{14}$ with respect to usual unbiased estimator $w_{z}^{2}$ along with the value of $\delta$ for which the PREs of the estimators $l_{3}$ to $l_{14}$ are maximum as given in [17].

It is observed from Tables 4 and 5 that all the estimators $l_{k}(k=15,16, \ldots, 20)$ which are the members of the proposed class of estimators $l$ performed better than the usual unbiased estimator $l_{1}=w_{z}^{2}$, ratio type estimators $l_{2}$ due to $[4,5]$ estimators $\left(l_{3}\right.$ to $\left.l_{8}\right)$ and [17] estimators $\left(l_{9}\right.$ to $\left.l_{14}\right)$, for $\varphi \in(-1.0,1.0)$.

\section{Conclusion}

In the present paper, we have suggested an improved class of estimators for population variance using the auxiliary information of nonconventional location parameters, nonconventional measures of dispersion, and their function with the coefficient of variation. The bias and mean square error (MSE) expressions of the proposed class of estimators are obtained and compared with the usual unbiased estimator, estimators in [4], [5], and [17]. We have also analyzed the performance of the proposed estimators by utilizing the data set of the known population and found that in convenient cases, the proposed estimators perform better than the other existing estimators. On comparison with the usual unbiased estimator, which is shown in Table 4, it is evident that upon increasing the values of $\varphi$ either negative or positive, the percent relative efficiency also increases. From Table 5, we observe that our estimators are more proficient than the existing ones. Hence, we strongly recommend that our proposed estimators perform better than the existing estimators for use in practical applications.

\section{Data Availability}

The data used to support the findings of this study are included within the article.

\section{Conflicts of Interest}

The authors declare that they have no conflicts of interest.

\section{References}

[1] A. K. Das and T. P. Tripathi, "Use of auxiliary information in estimating the finite population variance," Sankhya C, vol. 40, pp. 139-148, 1978.

[2] C. Kadilar and H. Cingi, "Ratio estimators for the population variance in simple and stratified random sampling," Applied Mathematics and Computation, vol. 173, no. 2, pp. 1047-1059, 2006.

[3] C. N. Bouza and M. Subzar, "Sub sampling rules for item non response of an estimator based on the combination of regression and ratio," Journal of King Saud Science, vol. 31, pp. 171-176, 2019.

[4] C. T. Isaki, "Variance estimation using auxiliary information," Journal of the American Statistical Association, vol. 78, no. 381, pp. 117-123, 1983.

[5] H. P. Singh, S. K. Pal, and R. S. Solanki, "Improved estimation of finite population variance using quartiles," Istatistik Journal of The Turkish Statistical Association, vol. 6, no. 3, pp. 166-121, 2013.

[6] H. P. Singh, S. K. Pal, and R. S. Solanki, "A new procedure for estimation of finite population variance using auxiliary information," Journal of Reliability and Statistical Studies, vol. 7, no. 2, pp. 149-160, 2014.

[7] M. Subzar, S. Maqbool, T. A. Raja, S. A. Mir, I. Jeelani, and M. A. Bhat, "Improved family of ratio type estimators for estimating population mean using conventional and nonconventional location parameters," Revista Investigacion Operacional, vol. 38, no. 5, pp. 499-513, 2017.

[8] M. Subzar, T. A. Raja, S. Maqbool, and N. Nazir, "New alternative to ratio estimator of population mean," International Journal of Agricultural and Statistical Sciences, vol. 12, no. 1, pp. 221-225, 2016.

[9] M. Subzar, T. A. Raja, S. Maqbool, S. A. Wani, A. B. Shikari, and N. Nazir, "Modified class of ratio estimators for population mean," International Journal of Agricultural and Statistical Sciences, vol. 14, no. 1, pp. 313-317, 2018.

[10] U. Shahzad, I. Ahmad, E. Oral, M. Hanif, and I. M. Almanjahie, "Estimation of the population mean by successive use of an auxiliary variable in median ranked set sampling," Mathematical Population Studies, vol. 88, 2020.

[11] U. Shahzad, I. Ahmad, I. Mufrah Almanjahie, N. H. Al - Noor, and M. Hanif, "A new class of L-Moments based calibration variance Estimators," Computers, Materials \& Continua, vol. 66, no. 3, pp. 3013-3028, 2021.

[12] U. Shahzad, M. Hanif, I. Sajjad, and M. Anas, "Quantile regression-ratio-type estimators for mean estimation under complete and partial auxiliary information," Scientia Iranica, vol. 21, 2020.

[13] M. Subzar, C. N. Bouza, S. Maqbool, T. A. Raja, and B. A. Para, "Robust ratio type estimators in simple random sampling using huber M estimation," Revista Investigacion Operacional, vol. 40, no. 2, pp. 201-209, 2019.

[14] M. Subzar, C. N. Bouza, and A. I. Al-Omari, "Utilization of different robust regression techniques for estimation of finite population mean in case of presence of outliers through ratio method of estimation," Revista Investigacion Operacional, vol. 40, no. 5, pp. 600-609, 2019.

[15] M. Subzar, A. Ibrahim Al-Omari, and A. R. A. Alanzi, "The robust regression methods for estimating of finite population 
mean based on SRSWOR in case of outliers," Computers, Materials \& Continua, vol. 65, no. 1, pp. 125-138, 2020.

[16] I. M. Almanjahie, A. I. Al-Omari, E. J. Ekpenyong, and M. Subzar, "Generalized class of mean estimators with known measures for outliers treatment," Computer Systems Science and Engineering, vol. 38, no. 1, pp. 1-15, 2021.

[17] R. S. Solanki, H. P. Singh, and S. K. Pal, "Improved ratio-type estimators of finite population variance using quartiles," Hacettepe Journal of Mathematics and Statistics, vol. 44, no. 3, pp. 747-754, 2015.

[18] M. N. Murthy, Sampling: Theory and Methods, Statistical Publishing Society, Calcutta, India, 1967. 